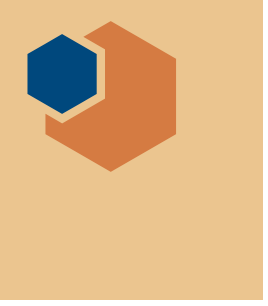

\title{
US Department of Energy launches grant program in quantum materials
}

\begin{abstract}
A fter years of lobbying and the eventual passage of a comprehensive funding bill, the first monies for a widespread federal effort to promote quantum information science (QIS) are now available to researchers. On January 10, the US Department of Energy (DOE) issued a request for funding applications totaling up to USD $\$ 625$ million over the next five years. This will go toward developing the National Quantum Initiative Program (NQIP). Final applications closed on April 10.

The NQIP was established by a bill signed into law on December 21, 2018, to "provide for a coordinated Federal program to accelerate quantum research and development for the economic and national security of the United States." Until recently, support for QIS on the national level has been relegated to
\end{abstract}

individual federal agencies at much smaller funding levels, according to Damon Dozier, Director of Government Affairs at the Materials Research Society (MRS), and Jeanie Lau, professor of physics at The Ohio State University. "Federal agencies identify research priorities annually and QIS has been one of those targeted priorities," Dozier says. "The agencies, for the most part, do a good job of listening to the community and trying to get a sense of what emerging topics are out there." For example, the National Science Foundation's (NSF) Enabling Quantum Leap has funded smaller teams, including a quantum materials foundry at the University of California, Santa Barbara.

This has been a long time coming. The government has invested in QIS since at least 1994, when Massachusetts Institute

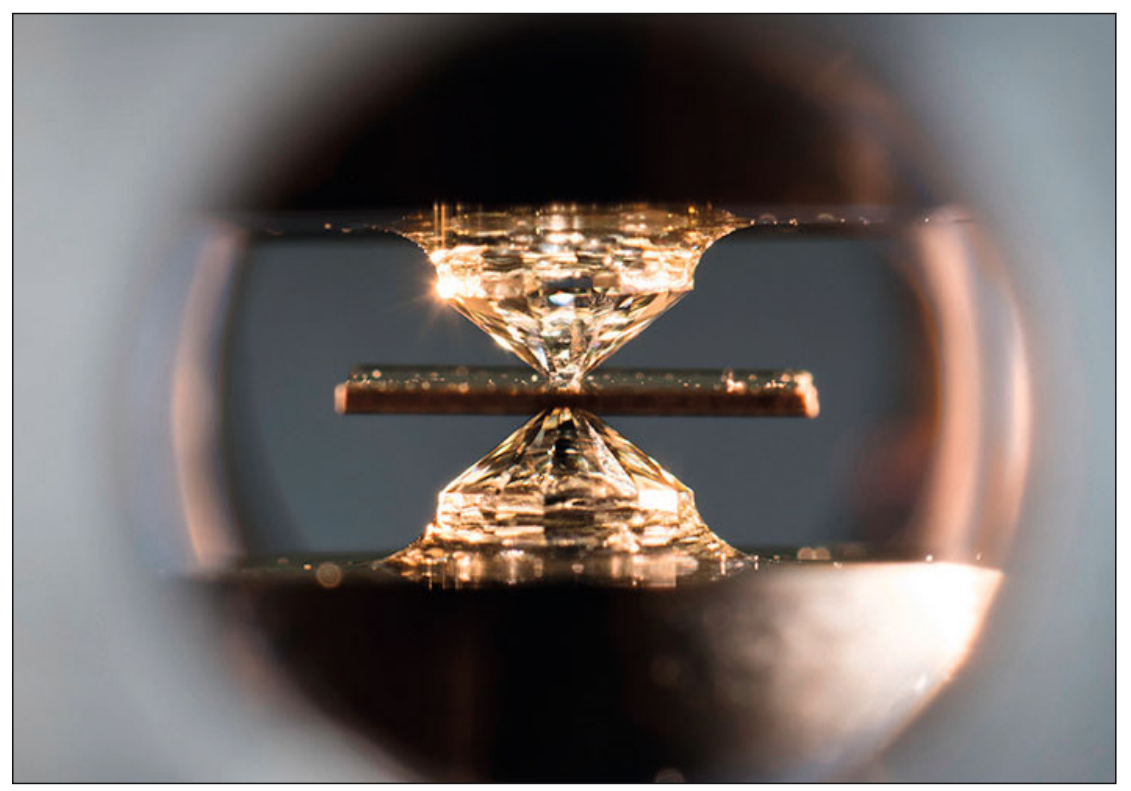

Diamond anvil cells compress and alter the properties of hydrogen-rich materials in Ranga Dias's laboratory of quantum phenomena at extreme conditions. Photo credit: J. Adam Fenster, University of Rochester.

of Technology professor of computer science Peter Shor devised a quantum algorithm to factor large numbers into primes while at Bell Laboratories, according to Jeremy Levy, professor of physics at the University of Pittsburgh and director of the Pittsburgh Quantum Institute. This was a key step in quantum cryptography; the first was the quantum key distribution protocol (cryptography) called BB84 created by Charles Bennett and Gilles Brassard in 1984. Levy himself was personally involved with the Defense Advanced Research Projects Agency's Quantum Information Science and Technology Program in 2000.

But a coordinated, long-term, plan had been lacking, Dozier says. "Getting that recognition from Congress and the White House is important." Making QIS a national priority is largely due to the lobbying efforts of scientists and science advocacy organizations, according to Dozier. Levy says, "The second quantum revolution has been coming for a long time, but it's started to heat up right now."

QIS promises to help answer questions that cannot be solved by conventional means. While standard computers rely on information coded as binary values (ones and zeroes), or bits, quantum computers store information as qubits. Qubits provide many more values by using intermediate and indefinite levels through quantum states.

The DOE solicitation is meant to start bringing that promise to fruition for the United States.

"Quantum information science will depend on the quantum properties of materials" to find practical, cost-effective ways to enable large-scale implementation, Levy says. He co-edited a 2013 article with James Eckstein of the University of Illinois about the intersectionality of quantum-based science 
and materials research for MRS Bulletin (doi:10.1557/mrs.2013.210). "It may mean discovering new materials, finding better materials synthesis and integration techniques for quantum computation and quantum communication, and exploring and exploiting new phenomena for topological quantum phenomena and quantum sensing," says Lau.

NQIP is, at least partially, a response to huge investments in QIS made by other countries. "Europe has decided to invest $€ 1$ billion over the next 10 years and China - significantly more than that," said Supratik Guha, a research director at Argonne National Laboratory (ANL) and former head of the Nanoscience and Technology Division and the Center for Nanoscale Materials at ANL (see MRS Bulletin, March 2018; doi:10.1557/ mrs.2018.46).The 2018 bill intends to bring the United States to the forefront of QIS through a 10-year plan to create a "QIS workforce pipeline."
The foundation of that plan is a series of QIS centers. So far, specifics on these centers are few but it is believed to follow the path of other research consortia. Dozier suspects the participating institutions will combine their interests, expertise, staff, equipment, facilities, and funding apparatuses for the QIS centers. "The [planned] centers will likely be affiliated with national laboratories," Levy says. As far as the numbers of centers to be created, that is "very much up for grabs." Also, unlike previously legislated centers, NQIP has no stipulation that specifically encourages institutions for underrepresented minorities, Dozier says.

Is the maximum allotment of USD $\$ 625$ million enough to compete against the rest of the world? "It is certainly a really good start," Lau answers. "I suspect that more investment will be made/needed to be truly competitive against Europe and China. There's a lot of talk of significantly ramping up investment," she adds. Though exactly how the monies will be divided has not yet been decided, Levy says. "The actual investment is not fully in place."

That future investment is planned in five-year tranches: the National Institute of Standards and Technology is to fund up to USD $\$ 400$ million; NSF is to fund up to USD\$50 million for each center (see MRS Bulletin, March 2020; doi:10.1557/ mrs.2020.76); and DOE is to fund up to USD $\$ 125$ million for each center, according to the legislation. But many a program has been derailed with money that was promised but not delivered.

MRS will urge Congress to provide more funds when it makes its semiannual visit to Capitol Hill, Dozier says. Lau cautions, "On the other hand, at this time of [a] tight budget situation, further investment in QIS might mean undercutting budgets in other muchneeded areas of basic and applied researchin my opinion this is a danger that funding agencies should strive to avoid."

Clinton Park
South African science ministries respond favorably to State of the Nation address by president

$\mathrm{B}$ lade Nzimande, who heads South Africa's Departments of Science and Innovation and of Higher Education, Science and Technology, supports President Cyril Ramaphosa's commitment on moving South Africa toward a low carbon growth trajectory.

The country's energy challenges have provided researchers with an opportunity to look differently at, for example, the use of hydrogen fuel cell technology (HFCT). The Minister confirmed that the National HFCT Research, Development and Innovation Strategy will continue to aim at stimulating innovation to further contribute toward the national electricity grid.

"We have also started rolling out demonstrations for the provision of electricity and mobility using platinum-based hydrogen fuel cells. These include the rolling out of fuel cells to a rural school in the North West," Minister Nzimande says.

The Minister welcomes the President's announcement of the establishment of a new University of Science and Innovation to be located in the city of Ekurhuleni, a major economic hub in the country. The university will be closely linked to driving high-impact and cutting-edge technological innovation with current and future industries, sectors, and firms to drive the frontiers of a future economy, the Minister says.

"We believe that this location, together with fresh new challenges arising from the revolution in science and technology in the third decade of the 21 st century, offers an opportunity for a new initiative to provide cuttingedge science and technology innovations across crucial areas such as data science, machine learning, artificial intelligence, block chain, robotics, and hydrogen-powered technologies including smart transportation and logistics systems," Nzimande says.

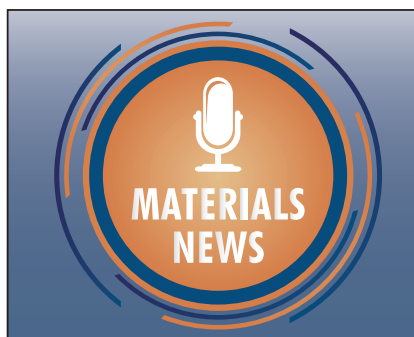

New episodes available at mrs.org/bulletin-podoast MRSBulletin PODBAST

Presenting breakthrough news and interviews with researchers on the hot topics of 3D bioprinting, artificial intelligence and machine learning, bioelectronics, perovskites, quantum materials, robotics, synthetic biology, and more. Listen Today! 